\title{
Intervention publique sur le développement de grappes industrielles au Québec
}

\author{
David Doloreux \\ Telfer School of Management, University of Ottawa \\ Diane-Gabrielle Tremblay \\ Télé-Université, Université du Québec à Montréal \\ Juan-Luis Klein \\ Département de Géographie, Université du Québec à Montréal \\ Steve Dionne \\ Centre de recherche sur le développement territorial (CRDT), Université du Québec à Rimouski \\ Tarek Ben Hassen \\ Doctorat en Études urbaines, Université du Québec à Montréal
}

Cet article décrit les conditions de développement de quatre grappes industrielles et les mécanismes d'insertion de ces dernières dans l'économie régionale. Les cas à l'étude sont les grappes de l'aérospatiale et du vêtement à Montréal, ainsi que de l'agroalimentaire et des sciences et technologies maritimes dans la région du Bas-Saint-Laurent. Les grappes industrielles sont généralement définies comme des arrangements d'entreprises, d'acteurs gouvernementaux, d'instances publiques locales et d'organisations intermédiaires concentrés sur un territoire local. Or, l'étude montre que la structuration d'une grappe met en ceuvre des acteurs et des processus fonctionnant à plusieurs échelles, du local jusqu'au global. Elle montre aussi l'importance d'un leadership sectoriel reconnu par les divers acteurs.

Mots clés : grappes industrielles, innovation, gouvernance, proximité, aérospatiale, vêtement, agro-alimentaire, sciences et technologies maritimes

\section{Public intervention in the development of industrial clusters in the province of Quebec}

This article describes the conditions for the development of four industrial clusters and the mechanisms of their integration in the regional economy. The case studies are the aerospace and apparel clusters in Montreal, and the agri-food and marine science and technology clusters in the Lower St. Lawrence region. Clusters are defined as arrangements of businesses, government actors, public authorities, and local intermediary organizations in a local area. However, the study shows that clusters are based on actors and processes operating at different scales, from the local to the global. It also shows the importance of the sectoral leadership acknowledged by the different stakeholders.

Keywords: clusters, innovation, governance, proximity, aerospace, clothing, agri-food, marine science and technology

\section{Introduction}

Face aux nouveaux impératifs imposés par le processus de mondialisation, les villes et les

Correspondence to/Adresse de correspondance: David Doloreux, Telfer School of Management, University of Ottawa, Ottawa, Ontario, Canada K1N 6N5. E-mail/Courriel: doloreux@telfer.uottawa.ca régions sont amenées à élaborer des initiatives et des stratégies destinées à stimuler de nouvelles formes d'activités économiques sur leurs territoires. À cette fin, la création de grappes industrielles constitue une stratégie fréquemment utilisée pour promouvoir l'innovation et l'entrepreneuriat aux échelles locales et régionales (Holbrook et Wolfe 2005; Guillaume

The Canadian Geographer / Le Géographe canadien 201 1, 55(4): 426-438

DOI: $10.1111 / \mathrm{j} .1541-0064.2011 .00386 . x$

(C) Canadian Association of Geographers / L'Association canadienne des géographes 
2008; Huggins 2008; Wolfe 2009; Doloreux et al. 2010). La notion de grappe industrielle peut être définie comme étant une forme d'agglomération d'entreprises interdépendantes et d'autres acteurs régionaux, d'un ou plusieurs secteurs, dans une aire géographiquement limitée (Porter 2003). Le postulat qui soutient ces stratégies est que les grappes industrielles peuvent s'influencer entre elles, et, en même temps, influencer positivement l'économie des régions en favorisant l'innovation, en augmentant la productivité régionale et en stimulant la création de nouvelles entreprises (Asheim et Gertler 2005).

Cet article décrit les conditions de développement de quatre grappes industrielles et les mécanismes d'insertion de ces dernières dans l'économie régionale, ainsi que le rôle joué par les gouvernements ou organismes publics dans la définition et le soutien de celles-ci. Les cas à l'étude sont les grappes de l'aérospatiale et du vêtement dans la région de Montréal, ainsi que celles de l'agroalimentaire et des sciences et technologies maritimes dans la région du Bas-Saint-Laurent. Les deux grappes de Montréal font partie d'un programme de développement de la Communauté métropolitaine de Montréal (CMM) qui a défini 15 grappes industrielles dans le cadre de sa politique de développement économique (CMM 2005). Les deux autres sont soutenues par des organismes locaux également. Il est entendu que les grappes, en tant que regroupement d'entreprises, d'acteurs de soutien et d'acteurs intermédiaires, peuvent fort bien exister sans le soutien public, mais ce qui nous intéresse dans cet article est de comprendre le rôle de l'action publique dans leur développement.

\section{La stratégie des grappes industrielles}

Bref retour sur la notion de grappe industrielle

Les grappes industrielles sont des regroupements géographiques d'organisations (entreprises et organisations publiques) œeuvrant dans des domaines d'activités similaires ou connexes. Elles ont été définies par Porter (2003) comme des concentrations géographiques d'entreprises interconnectées, de fournisseurs spécialisés, de prestataires de services d'entreprises fonctionnant dans des secteurs connexes et d'établissements associés, dans des domaines complémentaires. Pour cette définition, Porter s'appuie sur une conception des avantages compétitifs, qui, pour lui, ont un caractère local, proviennent de la concentration des connaissances et des savoir-faire, et relèvent de la présence d'institutions, de rivaux, d'entreprises partenaires et de consommateurs. Porter a introduit la notion de grappe industrielle comme étant une forme d'agglomération d'entreprises et d'acteurs régionaux, d'un ou plusieurs secteurs, interdépendants, dans une aire géographiquement limitée.

Selon cette perspective, les grappes industrielles soutiennent la croissance économique en offrant un environnement propice à l'innovation, à l'entrepreneuriat et au développement de nouvelles activités productives (Wolfe 2009). Les avantages procurés par les grappes industrielles seraient ainsi déterminés par la combinaison de différents facteurs orientés autour des effets bénéfiques engendrés par la proximité et la concentration géographique des acteurs. La proximité géographique des acteurs permettrait et faciliterait l'échange entre les entreprises, les sous-traitants, les établissements d'enseignement, les institutions de $\mathrm{R}$ et $\mathrm{D}$, etc. (Doloreux et al. 2010). À la proximité géographique, Tremblay et al. (2003) s'ajoute l'importance de la proximité relationnelle, c'està-dire des liens que nouent des entreprises, des instances d'intermédiation et la société locale. La proximité géographique et relationnelle d'entreprises et d'acteurs concentrés dans un territoire fournirait aux entreprises des externalités leur permettant d'innover et de croître (Cooke 2001; Maskell et Kébir 2006; Shearmur 2010). Ces externalités touchent la formation collective d'un bassin de main-d'œuvre qualifiée, l'attraction de fournisseurs et des connaissances partagées par des processus d'apprentissage collectif. Les liens sociaux permis par la proximité et les pratiques technologiques développées lors de processus d'apprentissage interactifs permettraient d'assurer une meilleure transmission du savoir et d'innover (Torre 2009). Le fonctionnement de tous ces éléments dépendrait, et en même temps serait générateur d'un environnement institutionnel local constitué de règles et normes agissant comme dispositifs cognitifs 
favorables au processus d'innovation (Doloreux 2004).

Mise en œuvre des grappes industrielles et rôle de l'intermédiation

Dans la documentation existante sur les grappes industrielles, on reconnaît que leur développement constitue un processus à long terme, et par conséquent les grappes peuvent se retrouver à des stades d'évolution différents. Ce processus est souvent animé par les instances gouvernementales, mais est fréquemment soutenu par la mobilisation des principaux acteurs dans la collectivité locale ou régionale (Klein et al. 2010). Parmi ces acteurs, on note la présence de ce que certains ont appelé des organismes intermédiaires ou organismes qui assument des fonctions d'intermédiation entre acteurs divers (Howell 2006).

L'une des principales fonctions des grappes, du moins celles qui sont constituées sur la base de politiques publiques, consiste à fournir le capital humain et social, de même que le capital financier et les ressources, au soutien des entreprises faisant partie de ces grappes industrielles, afin de favoriser l'innovation et le développement de la grappe. Outre les ressources humaines nécessaires à la production, les entreprises ont besoin d'autres formes de soutien pour maintenir leurs activités, ce qu'elles peuvent obtenir par le biais du capital social. En effet, c'est dans l'interaction avec d'autres organisations que se produisent des échanges de représentations et d'idées susceptibles d'induire des dynamiques innovantes au sein d'une grappe donnée (Julien 2005, 170).

Certains auteurs utilisent la métaphore de la colle pour illustrer comment ces liens et interconnexions peuvent agir, car ces liens peuvent renforcer les relations entre les membres d'un groupe ou d'une grappe. Lorsque l'information est riche (notamment l'information implicite ou tacite, et moins le savoir explicite et codifié), les interrelations et les échanges constituent d'excellents véhicules de transfert de l'information vers plusieurs acteurs; cela facilite la création de sens puisque la connaissance est partagée (Tremblay et Rousseau 2006). Dans ce contexte, la grappe est perçue comme un mode d'accès particulièrement efficace à l'interprétation de l'information et comme un bon environnement d'apprentissage pour les acteurs, dont les entreprises (Julien 2005, 171).

Julien (2005) souligne comment le capital humain et social contribue au soutien du développement des grappes industrielles. Au fond, les actifs collectifs que sont les normes, les conventions et la confiance faciliteront l'accessibilité et les échanges, stimulant ainsi la mobilisation des membres de la grappe. Une telle mobilisation devrait procurer de nombreux avantages, notamment en ce qui concerne le statut ou la réputation des principaux acteurs, ou encore au chapitre des ressources collectives ou du soutien accordé aux principaux projets. À son tour, l'attrait de ces avantages favorisera l'engagement des membres et leur participation.

\section{Les grappes industrielles et le développement des connaissances}

Dans le contexte de l'économie du savoir, la connaissance et l'apprentissage sont perçus comme des composants déterminants du développement économique et de la prospérité des régions et des nations; ils sont également considérés comme décisifs pour la compétitivité et le succès des entreprises dans leurs marchés. L'apprentissage est ainsi considéré comme l'un des principaux processus sociaux, celui qui procure l'accès au savoir. Bien qu'il soit possible pour les entreprises d'acquérir du savoir par des moyens traditionnels (achat de licence ou acquisition de brevet, etc.), cela ne procure pas l'accès à une base renouvelable de connaissances que fournit l'apprentissage. En effet, l'apprentissage est vu comme un processus continu qui permet aux individus, aux entreprises et aux territoires de changer et de s'adapter aux conditions ambiantes en perpétuelle évolution.

Ainsi, au sein des grappes industrielles, les facultés créatrices et l'innovation seraient favorisées par les interactions entre les agents socioéconomiques, l'apprentissage social dans un territoire donné et le développement du capital social. Par conséquent, les politiques qui visent leur création et leur mise en œuvre devraient s'appuyer sur des intermédiaires efficaces et reconnus, susceptibles d'offrir une diversité de sources de capital social, dont certaines contribueraient à obtenir le financement nécessaire 
au capital de démarrage, d'autres au soutien des entrepreneurs et le reste à favoriser l'émergence de nouvelles idées et de l'innovation.

Les principaux débats autour de la notion de grappe industrielle

De nombreux écrits ont réexaminé la stratégie des grappes industrielles en remettant en question certains de ces postulats (Martin et Sunley 2003). La première remise en question porte sur les effets de la proximité comme base d'une trajectoire de développement technologique et économique à l'échelle locale. D'après Echeverri-Carroll et Brennan (1999), Lundvall (2007), Torre et Rallet (2005), et Boschma (2005), la proximité géographique n'est pas un facteur déterminant pour comprendre et capter les dynamiques économiques et sectorielles. En effet, malgré l'importance des relations entre les différents acteurs d'une grappe industrielle (entreprises, décideurs, producteurs technologiques), ces relations ne résultent pas exclusivement de la proximité physique des différents acteurs. En d'autres termes, le simple fait de cohabiter sur le même territoire, soitil métropolitain ou non-métropolitain, n'est pas une condition suffisante pour que les différents acteurs entretiennent des relations.

De plus, il faut souligner que les acteurs peuvent développer des relations à une échelle globale, notamment dans des secteurs fortement internationalisés. Cette constatation pose la question de la place du local : dans quelle mesure le local est-il important pour le développement d'une grappe industrielle? Le territoire fut longtemps perçu comme un espace neutre, mais il est maintenant plutôt perçu comme un environnement déterminant pour les trajectoires d'innovation et de développement. Mais l'échelle pertinente pour que cette détermination se fasse sentir varie selon les secteurs et les types d'espace. L'organisation en grappe industrielle n'assure pas une trajectoire sans heurt; de ce fait, le concept de grappe doit être appliqué et soutenu différemment selon les milieux et selon les secteurs dans la mesure où le contexte global des secteurs d'activités économiques varie (Malerba 2005).

Par ailleurs, il a été montré empiriquement que l'espace local ne peut pas être autosuffisant, comme fournisseur d'intrants et de ressources critiques aux processus d'innovation des entreprises (Doloreux 2004; Simmie 2005). Il en est de même pour les dispositifs institutionnels de soutien à l'innovation mis en œuvre dans une perspective de développement régional et reposant sur des interventions publiques de niveaux géographiques variés (Howell 2006). Les réponses au soutien à l'innovation régionale passeraient ainsi par la mise en place d'une gouvernance multiscalaire impliquant des niveaux d'intervention à la fois suprarégionaux (nations), infrarégionaux (autorités locales/régionales, agglomérations), et transrégionaux (coordination interterritoriale) (Bramwell et al. 2008).

\section{Précisions méthodologiques}

Les études de cas n'ont pas été choisies dans une optique de représentativité conventionnelle, mais identifiées comme des grappes qui offrent une grande diversité de conditions économiques et sociales, reflétant des situations socioterritoriales diversifiées, couvrant des secteurs d'activités différents. Elles sont aussi de taille et de nature différentes, notamment en ce qui a trait à leur stade de développement. Néanmoins, le choix de ces cas a respecté les éléments de définition de la notion de grappe industrielle présentés dans la première section de ce texte, ainsi que la possibilité de vérifier certains éléments critiques que nous avons repérés dans la section précédente, ce qui permet une étude comparative féconde malgré la diversité des cas choisis.

La description des grappes a été centrée sur (1) les conditions d'émergence et le rôle joué par les gouvernements, les organismes publics et les politiques publiques dans le développement de la grappe; (2) le contexte géographique dans lequel s'insère la grappe; (3) les caractéristiques et les activités des organisations spécifiques à la grappe; (4) le mode de fonctionnement de la grappe. Ces éléments seront repris de façon systématique pour documenter les conditions de développement et les mécanismes d'insertion des grappes industrielles dans l'économie régionale.

Les informations traitant des quatre grappes industrielles s'appuient sur un nombre important d'entretiens réalisés auprès de dirigeants d'entreprises, de directeurs d'organismes 
intermédiaires ou de soutien, ainsi que d'intervenants régionaux. À Montréal, une vingtaine d'organismes intermédiaires intervenant dans la région ont été rencontrés, ainsi qu'une vingtaine d'entreprises pour chacun des secteurs, soit l'aérospatiale et le vêtement. Dans le Bas-Saint-Laurent, l'ensemble des organisations publiques et des organismes intermédiaires liés au développement de l'agrobiopôle et du secteur des sciences et technologies maritimes a été rencontré, ainsi qu'une dizaine d'entreprises pour chacun des secteurs. Les entretiens semi-directifs ont permis de retracer les principaux événements permettant de comprendre l'évolution des grappes étudiées à travers le temps, notamment en ce qui concerne le rôle des institutions et des politiques publiques dans leur trajectoire de développement. Les entretiens ont aussi été complétés par une recherche approfondie de documents de sources secondaires.

\section{Les études de cas}

\section{L'aérospatiale à Montréal}

L'aérospatiale est un secteur très important dans la région métropolitaine de Montréal, puisqu'on y trouve presque 50 pour cent des emplois du secteur au Canada et que la politique des grappes de la Communauté métropolitaine de Montréal (CMM) identifie cette grappe comme l'une des plus performantes et importantes dans sa politique de développement. À Montréal, le secteur de l'aérospatiale est organisé de manière plutôt hiérarchique et très structurée autour de quatre grands donneurs d'ordre, soit Bombardier Aéronautique, Bell Hélicoptères, Pratt\&Whitney et $\mathrm{CAE}$, et de quatre principaux organismes intermédiaires, soit l'Association québécoise de l'aérospatiale (AQA), le Comité pour l'adaptation de la main-d'œuvre de l'industrie aérospatiale du Québec (CAMAQ), le Consortium de recherche et d'innovation en aérospatiale du Québec (CRIAQ) et Aéro Montréal, nom reçu par l'organisation qui regroupe tous les acteurs de la grappe.

Le secteur compte quelque 250 entreprises et environ 40000 emplois. La masse salariale montréalaise représente environ 3,3 pour cent du total mondial, pour un chiffre d'affaires de quelque 12 milliards de dollars. Il s'agit du principal secteur d'exportation manufacturière du Québec. Le Québec compte 60 pour cent de la production du Canada dans ce secteur et plus de 70 pour cent de la $\mathrm{R}$ et $\mathrm{D}$ qui y est réalisée (Blum et Ebrahimi 2009).

Le secteur se caractérise par une nette dominance des quatre grands donneurs d'ordre (Ben Hassen et al., à paraître). La gouvernance se présente ainsi comme un arrangement entre ces donneurs d'ordre et les quatre principaux organismes intermédiaires du secteur. Les réseaux du secteur sont très fortement imbriqués entre eux, et très étroitement liés aux acteurs politiques, que ce soit à l'échelle locale (ville de Montréal et CMM), provinciale, ou fédérale, les trois nivaux de l'administration publique étant tous très attentifs aux demandes du secteur. De fait, compte tenu de l'importance économique du secteur, les gouvernements sont fortement à l'écoute des demandes exprimées par les entreprises.

La création d'Aéro Montréal, voire de la grappe de l'aéronautique, a de fait été initiée par les donneurs d'ordre du secteur et elle a sans doute pu prendre forme assez rapidement en raison de la concentration des entreprises du secteur autour des donneurs d'ordre (Klein et al. 2003; Zhegu 2007). En effet, en 2004, face aux nombreux défis observés dans le secteur (forte concurrence de nouveaux producteurs du Brésil et d'ailleurs, manque de main d'œuvre spécialisée, projets majeurs en suspens, dont celui de la Série C de Bombardier), les acteurs du secteur se sont mobilisés pour former le Comité aérospatial Québec qui, suite à des consultations, a abouti $\mathrm{au}$ lancement officiel d'Aéro Montréal, le 15 mai 2006. La grappe comme telle, en tant que concentration et regroupement d'entreprises et d'acteurs, existait déjà au moment de la création d'Aéro Montréal. Toutefois, la formalisation de celle-ci visait à bien y intégrer les entreprises plus petites et à diminuer les effets négatifs de certains conflits entre ce type d'entreprises et les plus grandes en raison de la concurrence pour les contrats et la main-d'œuvre.

Aéro Montréal fonctionne sur la base de cinq chantiers : innovation, visibilité, sous-traitance, main d'œuvre et productivité. ${ }^{1}$ Pour le chantier innovation, le CRIAQ s'est vu confier un rôle

${ }^{1}$ Les deux derniers n'étaient pas encore en fonctionnement au moment de notre enquête. 
important alors que l'AQA a pris en charge le chantier de la sous-traitance. On voit ainsi émerger des collaborations entre les divers organismes; même si on retrouve souvent les mêmes intervenants aux divers lieux. Cependant, malgré son rôle dans la création d'Aéro Montréal, la CMM n'est pas vue comme un leader par les acteurs de la grappe. Son rôle est davantage perçu comme un bailleur de fonds, apportant ainsi un soutien aux autres organismes en place. On peut donc considérer que dans ce cas, l'intervention publique a été importante pour assurer les interactions autres que productives entre les acteurs du secteur, pour développer les compétences locales afin d'assurer la continuité du dynamisme du secteur, pour faciliter la concertation au sein des acteurs et pour soutenir l'intermédiation entre les entreprises et les instances gouvernementales. L'intervention publique ici a été plus importante pour les PME, les grandes entreprises ayant bien sûr déjà leurs entrées directes auprès des gouvernements.

Les organismes intermédiaires agissent comme porte-parole du secteur auprès des autres acteurs en faisant la promotion de l'industrie et en identifiant les besoins des entreprises sur des questions aussi cruciales que la pénurie de maind'œuvre spécialisée, la nécessité de rapprocher les établissements d'enseignement locaux et les entreprises, ou encore l'importance du soutien étatique face à la concurrence internationale (Embraer au Brésil notamment). ${ }^{2}$

\section{Les sciences et technologies maritimes à Rimouski}

Les sciences et technologies maritimes constituent un secteur émergent dans la région du Bas-Saint-Laurent, plus particulièrement à Rimouski. Cette ville est le centre administratif du Bas-Saint-Laurent. Située à près de 500 kilomètres de Montréal, elle concentre une part importante de ses activités économiques dans les domaines de la santé et de l'éducation. Rimouski constitue un pôle important dans la région du Bas-du-Fleuve (Polèse et Shearmur

\footnotetext{
${ }^{2}$ Ce qui n'empêche pas par ailleurs certains équipementiers importants de collaborer aussi avec Embraer, ce qui permet de voir la situation à la fois de collaboration et de concurrence dans laquelle se situent les entreprises d'une grappe dynamique.
}

2002). En plus de divers services administratifs et d'éducation, y compris l'éducation supérieure, elle concentre des écoles, instituts et laboratoires dédiés aux sciences et techniques maritimes. Nous y retrouvons 14 entreprises qui emploient au total 153 personnes. Ces entreprises sont petites, la plupart comptant moins de 10 employés. Parmi ces entreprises, seuls le Groupe Trifide, ABK-Gaspésie et PESCA Environment emploient plus de 30 personnes, concentrant plus de 40 pour cent de l'ensemble de la maind'œuvre de ce secteur.

Ce secteur se décline en trois sous-secteurs. Le premier est celui de la biotechnologie marine. Il mise sur la valorisation des coproduits de transformation de la biomasse marine et sur la biodiversité pour les marchés nutraceutiques, cosméceutiques et pharmaceutiques. Il compte quatre entreprises et 54 employés. Le deuxième est celui des technologies maritimes et concerne les technologies développées pour les divers supports liés aux activités maritimes comme les géosciences, l'océanographie physique ainsi que la construction navale. Ce sous-secteur regroupe trois entreprises et 53 employés et la principale activité qui y est développée est la géomatique marine. Le troisième sous-secteur est celui des services-conseils en technologies maritimes qui offrent des services et des expertises spécialisés dans les différents domaines touchant les ressources, la science et les technologies maritimes. Ce sous-secteur comprend 8 entreprises et 46 employés. Les entreprises sont essentiellement concentrées à Rimouski et dans des villes relativement proches (Doloreux et Melançon 2006).

Au-delà des activités identifiées, les organisations publiques jouent un rôle important dans la structuration du secteur ainsi que le développement de nouvelles activités scientifiques et commerciales. À cet égard, la particularité est qu'un important réseau comprenant des institutions d'enseignement, des centres de recherche et de transfert technologique, ainsi que des organismes intermédiaires a été créé pour appuyer le développement de la grappe des sciences et technologiques maritimes. Parmi ces organisations, il y a les centres publics (Institut Maurice-Lamontagne, l'ISMER-Institut des sciences de la mer de Rimouski, l'Institut maritime du Québec, Innovation Maritime) et 
les centres privés à but non lucratif (Centre de recherche sur les biotechnologies marines, le Centre interdisciplinaire en développement de la cartographie des océans) (Doloreux et Melançon 2006). En l'absence d'une masse critique d'entreprises, ces organismes constituent un noyau incontournable dans les interactions structurelles et la nécessaire collaboration entre les différents acteurs du système de soutien et du système productif. Ils contribuent à la dynamique d'innovation et de création, ainsi qu'à la diffusion de connaissances dans le secteur des sciences et technologies maritimes.

L'importance de la présence d'organisations publiques n'est pas étrangère à la volonté des gouvernements fédéral et provincial, par le biais de différentes politiques et stratégies, de stimuler le développement des activités des sciences et technologies marines et d'une grappe maritime régionale, en s'appuyant notamment sur la présence, bien que limitée, d'activités économiques liées à l'estuaire du Saint-Laurent, de même que sur des infrastructures et des institutions publiques en place.

Les gouvernements, à travers différentes politiques (Stratégie d'Innovation Régionale et Plein cap sur la mer), avaient pour principal objectif le renforcement du dispositif d'accompagnement de ce secteur. Plus récemment, le développement du système productif constitue l'objectif principal de la stratégie de développement $d u$ créneau des ressources, sciences et technologies marines 2006-2011, élaboré dans le cadre du Programme ACCORD (Action Concertée de Coopération Régionale de Développement) par le gouvernement du Québec. Cependant, malgré le désir des gouvernements, le développement de la grappe en est encore à ses débuts. Le secteur est fragmenté et compte un nombre plus important d'acteurs publics que privés. L'absence d'une masse critique d'acteurs productifs privés, l'absence d'un marché régional et l'insécurité du financement des organisations de $\mathrm{R}$ et $\mathrm{D}$ constituent les principales contraintes qui limitent le développement de la grappe.

Le secteur des sciences et technologies marines confirme donc l'importance du soutien public, mais montre aussi qu'un tel soutien ne suffit pas pour garantir le développement d'une grappe industrielle. En l'absence d'acteurs productifs en nombre suffisant, il reste difficile de faire émerger une grappe industrielle digne de ce nom. Ceci a des conséquences importantes dans la mesure où les entreprises, bien qu'étant très actives en matière de $\mathrm{R}$ et $\mathrm{D}$, ont de la difficulté à commercialiser leurs produits et à les amener vers les marchés mondiaux. Par ailleurs, les organisations publiques qui doivent composer avec une insécurité dans le financement de leurs activités doivent se tourner vers l'extérieur pour trouver des contrats leur permettant de fonctionner, ce qui diminue la possibilité de faire profiter la région des activités de $\mathrm{R}$ et $\mathrm{D}$. Ceci crée une situation où le développement d'activités d'innovation et la mise en commun de ressources et d'échanges d'expertise entre la sphère privée et la sphère publique demeure faible. On peut conclure de ce cas que l'intervention publique et l'abondance d'organismes intermédiaires ne suffisent pas, et qu'il faut une certaine masse critique d'entreprises productives pour développer une véritable grappe industrielle.

\section{L'agrobiopôle à La Pocatière}

Le projet de l'agrobiopôle est une initiative réalisée à La Pocatière, une municipalité située dans la municipalité régionale de comté (MRC) de Kamouraska, à l'ouest du Bas-Saint-Laurent. Cette initiative a pour mandat de rassembler les éléments requis et de créer les conditions propices d'un projet territorial de développement autour de la création et de la mise en place d'un pôle majeur d'innovation dans les secteurs de l'agroalimentaire, de l'agroforesterie et de l'agroenvironnement. En ce sens, le projet s'inscrit également en continuité avec l'expérience historique peu commune des acteurs des nombreux établissements publics de ce milieu. Il vise à mettre en place un projet territorialisé de développement économique. (Doloreux et Dionne 2007).

Dans ce projet, le rôle de l'action publique est manifeste dans la définition de nouvelles opportunités pour la région et la capacité du milieu à générer localement la mise en place de nouvelles initiatives pour soutenir le développement économique. L'exemple de l'agrobiopôle est éloquent à cet égard. Ce projet a permis de mobiliser différents acteurs autour d'un projet commun. Le succès de ces mobilisations et de cette initiative repose en partie sur des complicités 
qui ne peuvent s'apprécier sans tenir compte de l'histoire qui caractérise le développement économique de La Pocatière et la dimension culturelle propre au milieu.

La Pocatière est une très petite ville située dans une région essentiellement rurale, localisée à une centaine de kilomètres de la ville de Québec. La Pocatière ne constitue certes pas un pôle important parmi les centres urbains qui se déploient sur l'ensemble du territoire bas-laurentien et dont les plus importants sont Rimouski et Rivière-du-Loup. Dans sa MRC, elle joue cependant le rôle de centre institutionnel, administratif et commercial local, particulièrement dans les domaines de la santé et de l'éducation. Plus encore, en raison de la présence depuis plus de 35 ans d'une grande entreprise manufacturière, Bombardier, elle fait également office de centre manufacturier incontournable : le plus important de tout l'Est-du-Québec.

Le projet d'agrobiopôle a été lancé en 2002 par le Centre de développement bioalimentaire du Québec (CDBQ) en collaboration avec les différents intervenants de développement économique et agroalimentaire de la région, notamment avec l'Institut de technologie alimentaire (campus La Pocatière), la Corporation de développement économique de La Pocatière, le Centre local de développement et la Société d'aide au développement de la collectivité de Kamouraska (Doloreux et Dionne 2007). En cherchant à valoriser la maîtrise et la circulation de l'information dans le but de favoriser l'innovation et l'entrepreneuriat, le projet a comme objectif principal de faire en sorte que La Pocatière devienne un pôle majeur d'innovation au Québec dans les secteurs de l'agroalimentaire, l'agroforesterie et l'agroenvironnement.

Un autre objectif principal du projet d'agrobiopôle est de contribuer au développement de l'activité économique régionale et à la création d'emplois. En raison des acteurs associés au projet et de la présence d'une certaine masse critique scientifique, technologique et industrielle dans la région pocatoise, l'agrobiopôle ambitionne d'assumer également une vocation régionale, c'est-à-dire de favoriser et de soutenir dans une perspective de développement durable les innovations technologiques, industrielles et commerciales des secteurs visés. Le projet s'inscrit dans le contexte des besoins actuels en matière de développement et d'innovation liés à l'agroalimentaire, l'agroforesterie et l'agroenvironnement. Il vise la diversification de l'économie régionale dans ces secteurs, le développement et le soutien des entreprises, le recrutement d'une main-d'œuvre qualifiée, et l'accroissement des activités de transformation.

Le fonctionnement de l'agrobiopôle est assuré par un animateur travaillant en association étroite avec le CDBQ alors que divers partenaires locaux et régionaux sont interpellés pour l'articulation et l'offre de différentes compétences et connaissances s'inscrivant dans sa mission et ses différentes fonctions. Pour épauler le fonctionnement de l'Agrobiopôle, un comité constitué des principaux acteurs du milieu pocatois a été mis en place (CDBQ, ITA-Institut de technologie agroalimentaire, CDÉ-Corporation de développement économique, CLD-Centre local de développement du Kamouraska et SADCSociété d'aide au développement de la collectivé du Kamouraska). Ce comité a pour mandat de favoriser la mise en commun de l'expertise des différents partenaires pour orienter, offrir des services et appuyer les initiatives porteuses en matière de développement de nouvelles entreprises.

$\mathrm{Au}$ moment de notre enquête, on trouvait sept entreprises associées à l'agrobiopôle. Bien que ce nombre soit faible, la présence de l'incubateur apparaît concrètement comme l'une des pièces maîtresses du dispositif. Dans ce projet de développement, l'Incubateur bioalimentaire (IBA), équipement géré par le $\mathrm{CDBQ}$ en partenariat principal avec l'ITA, apparaît comme une organisation de soutien importante. En plus de matérialiser les efforts de ce milieu pour le redéveloppement de sa vocation agroalimentaire et agroenvironnementale, c'est-à-dire le projet territorial de rendre à ce milieu une vocation qu'il a déjà eue lorsque La Pocatière était un centre important de rayonnement agricole et agronomique à l'échelle du Québec, la présence de l'incubateur permettra l'accueil d'entreprises et favorisera la création de nouvelles entreprises.

L'agrobiopôle n'a cependant pas de correspondance matérielle à proprement parler. Il ne correspond pas à un bâtiment, à une installation physique ou encore à une organisation dotée d'un personnel propre. L'agrobiopôle est 
plutôt la formalisation d'un réseau d'acteurs et de partenaires du milieu dont l'ambition est de faire ressortir et de mettre en commun de manière stratégique les compétences et les expertises de chacun au profit de l'ensemble, un outil pour consolider de nouveaux partenariats entre les différents acteurs partageant une vision commune du développement économique et technologique dans la région. L'agrobiopôle mise également sur le développement de réseaux de coopération et d'alliances stratégiques avec des sites d'excellence complémentaires à l'échelle du Québec, du Canada et de l'international.

Contrairement au cas de la grappe Textile et vêtement à Montréal et à celui des sciences et technologies maritimes à Rimouski, l'agrobiopôle est une initiative issue de la mobilisation des institutions et organisations du milieu. La composante entrepreneuriale faible (seulement 7 entreprises) est compensée par la mobilisation des acteurs locaux autour de projets communs, ce qui a permis de mettre en place des structures communes pour générer de nouvelles connaissances que de nouvelles entreprises pourront valoriser.

L'intervention publique a clairement pour objectif ici de faire émerger la grappe industrielle, de la soutenir et d'en faire l'élément clé du développement de la région. Le fait qu'il y ait peu d'autres activités importantes dans la région devrait favoriser la collaboration, mais il faudra sans doute aussi tenter d'attirer davantage d'entreprises ou de retenir sur le territoire les étudiants qui sortent de l'organisme de formation (ITA). Ce cas illustre encore le fait que l'intervention publique ne suffit pas à créer une grappe et à la dynamiser puisqu'il faut encore développer les entreprises qui puissent matérialiser les résultats de la collaboration.

\section{Le vêtement à Montréal}

À Montréal, le secteur du vêtement a historiquement été très important. Il s'agit d'une des spécialisations industrielles de la métropole, notamment en termes d'emploi. Au début des années 2000, le vêtement employait environ quarante mille travailleurs, ce qui constituait 40 pour cent de l'ensemble de l'emploi dans ce secteur au Canada. Ce secteur s'est concentré dans un quartier connu traditionnellement comme le «quartier de la guenille», dont aujourd'hui les acteurs locaux essaient d'améliorer l'image en adoptant le nom de «Cité de la mode» (Klein et al. 2007a, 2007b). Ce quartier se caractérise par la prédominance d'un grand nombre de petites entreprises, souvent assez fragiles, bien que quelques designers aient récemment rejoint le quartier, améliorant ainsi à la fois son image et sa viabilité.

Le secteur est en profonde transformation, particulièrement depuis la levée des dernières barrières tarifaires en janvier 2005. À cause de la concurrence internationale et du besoin de s'adapter aux conditions de commercialisation imposées par les grandes surfaces (Wal-Mart, Sears, La Baie), les entreprises tendent à déplacer la fabrication vers des pays qui offrent de meilleures conditions de rentabilité (Chine, Viêt Nam, Bangladesh). Ceci se traduit par une forte diminution du nombre d'entreprises et par des pertes d'emploi, ce qui provoque des réactions de la part des acteurs publics et des organisations représentatives du secteur (Klein et al. 2007a).

Le secteur du vêtement se divise en trois principaux sous-secteurs : fabrication, mode et habillement. Il comprend un grand nombre d'entreprises, en général de faible taille, et aussi des établissements de formation de niveau collégial et universitaire. Tout en demeurant important malgré les changements, le secteur est plutôt fragmenté, la concertation et la collaboration y étant peu développées. La seule exception se trouve dans le domaine de la fourrure qui constitue une grappe très innovatrice favorisée par les interrelations productives et le leadership du Conseil canadien de la fourrure, un organisme à but non lucratif localisé à Montréal (Klein et al. 2007b).

Il est difficile dans le cas du vêtement de parler d'une grappe mature, voire même d'une grappe. Or la CMM a planifié le développement d'une grappe désignée comme Textiles et vêtement, qu'elle voit comme une grappe de compétition, au même titre que l'aérospatiale. La plupart des entreprises, surtout celles concentrées dans la Cité de la mode, sont caractérisées par une très faible innovation (la fabrication), alors que les entreprises œuvrant dans d'autres domaines (textiles intelligents, vêtements de plein air, designs innovants, 
productions éthiques, etc.), qui affichent une capacité d'innovation plus forte, sont moins nombreuses et sont plutôt dispersées sur le territoire. De plus, le secteur est caractérisé par l'absence d'un leadership et d'un acteur clé pouvant rassembler tous les acteurs productifs et institutionnels.

Les relations entre les acteurs sont caractérisées par de nombreux conflits et par ce que l'on peut qualifier de culture de «clans». Les acteurs collaborent peu et, comme nous l'indiquent nos entrevues, plusieurs ne comptent pas le faire dans le cadre de la grappe que la CMM souhaite mettre en place. Déjà le choix d'inclure le textile dans la grappe est fortement contesté par les organismes que nous avons rencontrés, qui considèrent que ces deux secteurs sont trop opposés pour être regroupés. De ce fait, on peut penser qu'il y aurait plutôt des grappes productives autour de certains produits, comme la fourrure, ou autour de certaines fonctions, comme le design (Tremblay et al. 2009; Klein et al. 2010). La stratégie de concertation privilégiée par la CMM est ici inefficace. Malgré le grand nombre d'entreprises, celles-ci sont peu intégrées au plan productif, et le secteur manque visiblement d'organismes intermédiaires efficaces capables de concerter l'ensemble des acteurs.

Pourtant, on pourrait imaginer que les divers sous-secteurs favorisent la circulation de connaissances de l'un vers l'autre, par exemple, des textiles intelligents vers les vêtements de plein air ou encore les vêtements de design haut de gamme. Alors que l'on affirme que le secteur doit évoluer vers le design ou les productions éthiques, par exemple, s'il veut conserver des emplois face à la concurrence des pays à bas coûts de main-d'œuvre, les designers sont plutôt individualistes et ne recherchent pas tous le réseautage, bien que quelques cas, comme le Lab Créatif, aient permis d'illustrer les apports potentiels du réseautage et de la collaboration dans ce secteur (Klein et al. 2007a).

Nous sommes donc ici en présence d'une grappe émergente, qui doit évoluer vers des niches spécialisées pour assurer son développement, voire sa survie, tant la concurrence internationale est dure, mais que l'intervention publique n'a pas su reconnaître et soutenir correctement; le vêtement et le textile sont en fait deux secteurs qui cadrent mal dans un concept global, homogénéisant, d'échelle métropolitaine, tel que conçu par la CMM. Ce cas illustre aussi une situation opposée à celle des sciences et technologies maritimes à Rimouski. En l'absence d'organismes intermédiaires forts et reconnus, la présence d'un grand nombre d'entreprises productives ne permet pas la création d'une grappe. La stratégie publique devrait alors délaisser l'approche du grand secteur Textile et vêtement et se concentrer sur les véritables cadres de coopération productive existants, en s'appuyant sur les organisations susceptibles de soutenir la concertation des acteurs. À cet égard, les acteurs du développement économique communautaires de soutien à l'entrepreneuriat se sont parfois révélés efficaces, comme le montre le cas du Lab Créatif (Klein et al. 2007a).

\section{Discussion}

La description des quatre grappes industrielles démontre la forte présence de l'acteur public local représenté, dans la région de Montréal, par la Communauté métropolitaine de Montréal avec sa politique des grappes, et au Bas-SaintLaurent, par la gestion du Programme ACCORD élaboré par le ministère du Développement économique, de l'Innovation et de l'Exportation (MDEIE) pour appuyer le développement des régions québécoises. Elle montre, par ailleurs, l'importance que prend la concertation entre les acteurs dans la mise en œuvre de stratégies visant la création de grappes industrielles, aux dépens de la concertation entre et avec les entreprises.

Dans l'ensemble, chaque cas illustre une dynamique d'acteurs différente, mais les quatre cas nous donnent à voir le spectre d'acteurs contribuant au développement local au Québec. Ces acteurs comprennent essentiellement des acteurs gouvernementaux, des instances publiques locales, des entreprises et des organisations intermédiaires non gouvernementales. Dans chaque cas, les combinaisons de leadership des acteurs, d'arrimages, ainsi que d'intervention publique, que ce soit à travers les organisations gouvernementales ou les instances publiques locales, sont différentes. 
L'entreprise privée domine clairement dans l'aérospatiale, mais le rôle des organisations intermédiaires et des gouvernements y est crucial. Dans le cas des sciences et technologies maritimes à Rimouski, il y a présence d'organisations intermédiaires et des organisations gouvernementales, mais une faible présence entrepreneuriale. Dans le cas de La Pocatière, il s'agit surtout d'organisations intermédiaires locales avec un fort potentiel d'entrepreneuriat, mais qui n'est pas encore matérialisé, et dans le cas du vêtement, nonobstant le nombre d'entreprises, il s'agit d'un projet d'une instance publique locale, sans soutien clair que ce soit du gouvernement, des organisations intermédiaires, ou des entreprises. Au contraire, ces acteurs s'arriment et interagissent, mais sur d'autres projets qui ne coïncident pas nécessairement avec le projet de grappe, et que celui-ci n'est pas capable d'intégrer.

Il est intéressant de souligner que les échanges d'information semblent plus forts dans les domaines de l'aérospatiale et de l'agrobiopôle, essentiellement parce que ces deux secteurs sont caractérisés par une concertation profondément ancrée historiquement. Dans ces deux cas, l'interrelation entre les acteurs est antérieure au projet de grappe. Dans les deux autres cas, la concertation est récente, comme dans le cas des technologies marines, voire à construire, comme dans le cas du vêtement. Dans ces deux derniers cas, la grappe est un projet plutôt politique.

On pourrait croire que les grappes qui se développent dans un cadre métropolitain regroupent plus d'acteurs et bénéficient d'une infrastructure mieux développée, ce qui devrait leur permettre de générer un dynamisme endogène plus fort que dans les cas des régions non métropolitaines. Ceci ne s'avère que partiellement. La présence de plus d'acteurs peut aussi devenir un problème dès lors qu'il n'y a pas une tradition de collaboration, comme le montre le cas du vêtement. Par ailleurs, le cas de La Pocatière montre qu'il est possible de développer du dynamisme en région non métropolitaine, pour autant qu'il y ait un leadership endogène fort et reconnu. Du côté de Rimouski, l'absence d'un tel leadership limite la capacité de la grappe à se développer.

\section{Conclusion}

Les quatre cas étudiés illustrent bien le fait que le développement des grappes industrielles peut se présenter de manière très différente, bien que nombre d'écrits aient pu laisser penser qu'il y avait une «recette unique» pour créer une grappe industrielle. En effet, les cas étudiés montrent qu'il n'existe pas un modèle ou un ensemble unique de facteurs qui permettent d'expliquer de façon satisfaisante le développement d'une grappe. Ils montrent aussi que les dynamiques spatiales se combinent avec des dynamiques institutionnelles sectorielles.

Certains secteurs, comme l'aéronautique, présentent un réseau productif très concentré, très intégré verticalement, qui s'est rapidement inséré dans la politique des clusters de la CMM, laquelle n'est venue que coiffer des collaborations de production et de recherche existant déjà entre les différents types d'acteurs. Par contre, d'autres secteurs, comme le vêtement, regroupent une multitude de PME; dans ce cas, la collaboration et la concertation y sont clairement plus difficiles à cause de l'absence d'intégration des entreprises et de l'absence d'un leadership sectoriel fort. On retrouve par contre plusieurs organisations de sous-secteurs dans le vêtement, mais l'organisation d'une grappe unique ne leur permet pas de s'affirmer. Ceci montre qu'une région métropolitaine comme Montréal peut être traversée par des dynamiques entrepreneuriales très divergentes qui relèvent davantage des trajectoires institutionnelles sectorielles que des attributs territoriaux.

De même, les deux cas situés en région non métropolitaine, soient l'agrobiopôle et les sciences et technologies maritimes, présentent aussi des situations très différentes. Dans un cas (agrobiopôle), il s'agit principalement d'un réseau sectoriel localisé, sans organisme politique pour soutenir son développement. Dans l'autre, c'est un ensemble d'organisations régionales qui se concertent autour de certains acteurs clés.

La documentation des cas a permis de souligner quatre éléments en lien avec les conditions de développement de quatre grappes industrielles et les mécanismes d'insertion de ces dernières dans l'économie régionale. Premièrement, l'émergence et le développement 
d'une grappe ne se décrètent pas, contrairement à ce que laissent parfois penser les écrits de nature normative. On peut bien sûr mettre en place des politiques favorisant les échanges et le développement d'une grappe, mais on ne peut assurer que les échanges d'information et de connaissances se produiront effectivement, encore moins qu'ils produiront de l'innovation. Deuxièmement, on constate que le développement d'une grappe relève de processus institutionnels ancrés dans le territoire et non pas uniquement du fait d'être proche, ces processus étant multiples surtout dans les milieux urbains complexes. Troisièmement, on constate l'importance des dynamiques sectorielles et des leaderships qui s'y développement, qu'ils soient liés à l'entreprise, comme dans l'aérospatiale, ou aux organisations, comme pour l'agrobiopôle. Quatrièmement, le développement des grappes constitue un processus à long terme, qui relève du développement d'arrangements d'acteurs ancrés dans le territoire.

Pour conclure, il est important de souligner que la présence d'un leadership fort d'un ou quelques acteurs centraux susceptibles d'établir des consensus est cruciale pour le développement d'une grappe. Mais il est clair aussi qu'une concertation doit s'établir entre l'ensemble des acteurs. Les coopérations entre entreprises prennent du temps pour se développer et les stratégies publiques devraient tenir compte de cette réalité.

Si l'intervention publique ne suffit pas à développer une grappe, elle peut sans doute y contribuer. Des associations sectorielles ou des organismes publics peuvent jouer un rôle important, pour autant que ce rôle soit reconnu et accepté par les entrepreneurs (Klein et al. 2007a; Tremblay et al. 2009). Ceci permet de rappeler qu'une grappe industrielle constitue l'ancrage local ou régional d'un réseau sectoriel, ce qui oblige à tenir compte des logiques sectorielles dans l'analyse.

La notion de grappe fait aussi référence à la proximité entre entreprises et organisations et à l'idée que cette proximité puisse développer les liens, accélérer les flux d'information et de connaissances, et favoriser l'innovation. De fait, on peut dire que les projets localisés au Bas-Saint-Laurent avaient clairement pour objectif de transformer le profil économique de la région et de transformer les tendances lourdes de développement qui caractérisent les régions périphériques. En milieu métropolitain, l'impact de la collaboration vise à assurer le succès d'un secteur, en espérant qu'il ait un effet d'entraînement suffisamment important pour accroître la performance de la ville entière, voire de la région. On constate cependant que les choses ne s'enclenchent pas automatiquement, peu importe le type de région. En fait, les grappes les plus dynamiques constituent des configurations productives locales, qui incluent des organisations locales, mais aussi régionales et nationales et qui sont appuyées par les gouvernements nationaux.

\section{Remerciements}

Les auteurs remercient l'éditeur, Marius Thériault, ainsi que les trois évaluateurs anonymes pour les commentaires et suggestions apportés pour l'amélioration de l'article. Nous tenons à remercier le Conseil de recherche en sciences humaines du Canada (CRSH) et le programme The Innovations Systems Research Network (ISRN), coordonné par David Wolfe et Meric Gertler, qui a financé une bonne partie des travaux.

\section{Références}

Asheim, B. T. et M. S. Gertler. 2005. Regional innovation systems and the geographical foundations of innovation. The Oxford Handbook of Innovation, dir. J. Fagerberg, D. Mowery, et R. Nelson. London, UK: Oxford University Press, 291-317.

Ben Hassen, T., J. -L. Klein, et D. -G. Tremblay. à paraître. Building local nodes in a global sector: Clustering within the aeronautics industry in Montreal. Le Géographe canadien.

Blum, G., et M. Ebrahimi. 2009. Le secteur aéronautique au Québec. Bulletin Vision 2(1): octobre 2009. http://www. esg.uqam.ca/vision/2009/octobre/rech_aeronatique.php.

Boschma, R. 2005. Proximity and innovation: A critical assessment. Regional Studies 39(1): 61-74.

Bramwell, A., J. Nelles, et D. A. Wolfe. 2008. Re-locating the local: Urban governance and the scalar debate. Paper presented at the Annual Meeting of the Canadian Political Science Association, June 3-6, in Vancouver, BC.

CMM (Communauté Métropolitaine de Montréal). 2005. Plan de développement économique. Montréal, QC: CMM. http://www.cmm.qc.ca/index.php?id=201.

Cooke P. 2001. Regional innovation systems, clusters, and the knowledge economy. Industrial and Corporate Change 10(4): 945-974. 
Doloreux, D. 2004. Regional innovation systems in Canada: A comparative study. Regional Studies 38(5): 479-492.

Doloreux, D., et S. Dionne. 2007. Évolution d'un système local d'innovation en région rurale. Le cas de La Pocatière dans une perspective historique (1827-2005). Rimouski, QC: Éditions du GRIDEQ et du CRDT.

Doloreux, D., et Y. Melançon. 2006. Le pari ambitieux du cluster maritime du Québec: État de la situation et politiques publiques. Géographie, Économie, Société 8(4): 467-480.

Doloreux, D., Y. Melançon, et G. Devillet. 2010. Programmes et actions publiques pour stimuler l'innovation régionale dans la compétitivité mondiale. Canadian Public Administration 53(3): 573-601.

Echeverri-Carroll, E., et W. Brennan. 1999. Are innovation networks bounded by proximity? Innovation, Networks, and Localities, dir. M. Fischer, L. Suarez-Villa, et M. Steiner. Berlin, Allemagne: Springer-Verlag, 28-49.

Guillaume, R. 2008. Des systèmes productifs locaux aux pôles de compétitivit é: Approches conceptuelles et figures territoriales du développement. Géographie, Économie, Société 10(3): 295-309.

Holbrook, A., et D. Wolfe. 2005. The innovation systems research network (ISRN): A Canadian experiment in knowledge management. Science and Public Policy 32(2): 109118.

Howell, J. 2006. Intermediation and the role of intermediaries in innovation. Research Policy 35: 715-728.

Huggins, R. 2008. The evolution of knowledge clusters: Progress and policy. Economic Development Quarterly 22(4): 277-289.

Julien, P. -A. 2005. Entrepreneuriat régional et économie de la connaissance. Une métaphore des romans policiers. Québec, QC: Presses de l'Université du Québec.

Klein, J. -L., D. -G. Tremblay, et J. -M. Fontan. 2003. Systèmes locaux et réseaux productifs dans la reconversion économique: Le cas de Montréal. Géographie, Économie et Société 5(1): 59-75.

Klein, J. -L., D. -G. Tremblay, et D. -R. Bussières. 2007a. L'industrie de la mode dans l'alternative: Les perspectives d'une reconversion annoncée. Organisation et territoires 6(1): 89-95.

Klein, J. -L., D. -G Tremblay, J. -M. Fontan, et N. Guay. 2007b. The uniqueness of the Montreal fur industry in an apparel sector adrift: The role of proximity. International Journal of Entrepreneurship and Innovation Management 7(2,3,4,5): 298-319.

Klein, J. -L., D. -G. Tremblay, et D. -R. Bussières. 2010. Social economy-based local initiatives and social innovation:
A Montreal case study. International Journal of Technology Management 51(1): 121-138.

Lundvall, B. -A. 2007. National innovation systems-Analytical concept and development tool. Industry \& Innovation 14: 95-119.

Malerba, F. 2005. Sectoral systems: How and why innovation differs across sectors. The Oxford Handbook of Innovation, dir. J. Fagerberg, D. C. Mowery, et R. R. Nelson. Oxford, UK: Oxford University Press, 380-406.

Martin, R., et P. Sunley. 2003. Deconstructing clusters: Chaotic concept or policy panacea? Journal of Economic Geography 3: 5-35.

Maskell, P., et L. Kébir. 2006. What qualifies as a cluster theory? Cluster and Regional Development: Critical Reflections and Explorations, dir. B. T. Asheim, P. Cooke, et R. L. Martin. London, UK: Routledge, 30-49.

Polèse, M., et R. Shearmur. 2002. La périphérie et l'économie du savoir. Montréal, QC: INRS-Urbanisation, Culture et Société et Moncton, NB: Institut Canadien de Recherche sur le Développement Régional.

Porter, M. E. 2003. The economic performance of regions. Regional Studies 37(6-7): 549-578.

Shearmur, R. 2010. Space, place, and innovation: A distance-based approach. Le Géographe canadien 54(1): 46-57.

Simmie, J. 2005. Innovation and space: A critical review of the literature. Regional Studies 39: 789-804.

Torre, A. 2009. Retour sur la notion de proximité géographique. Géographie, Économie, Société 11: 63-75.

Torre, A., et A. Rallet. 2005. Proximity and localization. Regional Studies 39: 47-60.

Tremblay, D. -G., J. -L. Klein, et J. -M. Fontan. 2009. Initiatives locales et développement socioterritorial. Québec, QC: Presses de l'Université du Québec.

Tremblay, D. -G., J. -L. Klein, J. -M. Fontan, et S. Rousseau. 2003. Proximité territoriale et innovation: Une enquête sur la région de Montréal. Revue d'économie régionale et urbaine 5: 835-852.

Tremblay, D. -G., et S. Rousseau. 2006. The Montreal multimedia sector: A cluster, an "innovative milieu", or a simple colocation? Canadian Journal of Regional Science 28(2): 299-327.

Wolfe, D. 2009. 21st Century Cities in Canada. The Geography of Innovation. Ottawa, ON: The Conference Board of Canada.

Zhegu, M. 2007. La coévolution des industries et des systèmes d'innovation: L'industrie aéronautique. Thèse de doctorat, Université du Ouébec à Montréal. 